\title{
Factors Influencing the Suitability of Thermal Methods for Stress Analysis of NiTi Shape Memory Alloys
}

\author{
J. Eaton-Evans ${ }^{1 a}$, J. M. Dulieu-Barton ${ }^{2 b}$, E. G. Little ${ }^{3 c}$ and I. A. Brown ${ }^{3 d}$ \\ ${ }^{1}$ Department of Engineering Sciences, University of Oxford, UK. \\ ${ }^{2}$ School of Engineering Sciences, University of Southampton, UK. \\ ${ }^{3}$ Department of Mechanical and Aeronautical Engineering, University of Limerick, Ireland. \\ ajames.Eaton-Evans@eng.ox.ac.uk, bjanice@ship.soton.ac.uk, ${ }^{\mathrm{c} E d w a r d . L i t t l e @ u l . i e, ~}{ }^{\mathrm{d}}$ lan.Brown@ul.ie
}

Keywords: NiTi alloy, superelastic, shape memory, thermoelastic stress analysis

\begin{abstract}
The material assumptions made to facilitate Thermoelastic Stress Analysis (TSA) are linear elasticity, material homogeneity and isotropy, and mechanical properties that are independent of temperature. The unusual shape memory and superelastic properties of near equiatomic NiTi alloys complicate the application of any experimental stress analysis technique, and in the case of TSA, make these assumptions invalid. This paper describes a detailed analysis conducted to characterise the material properties of NiTi shape memory alloys and to identify loading conditions suitable for quantitative stress analysis using TSA. The mechanical behaviour of the material in three distinct regions is considered and the suitability of each region for TSA is discussed. It is shown that the thermoelastic response is dependent on the mean stress when tested at room temperature in the pre-martensitic phase, due the presence of an intermediate R-phase. Theoretical calculations are used to confirm that this effect is related to the high temperature dependence of the material's Young's modulus.
\end{abstract}

\section{Introduction}

In Thermoelastic Stress Analysis (TSA) [1] it is assumed that the test material is homogenous and isotropic, that quasi-adiabatic conditions can be achieved by cyclically loading the specimen, the loading remains within the linear elastic range of the material, the mechanical properties of the material are not dependent on temperature and that the ambient temperature remains constant during testing [2, 3]. These assumptions are necessary for an accurate analysis; however an irregularity in the thermoelastic response that results from a failure to comply with one of the assumed conditions can be used to infer additional information about the loaded component. For example, it has been shown [4] that areas of residual stresses can be identified by examining variations in the thermoelastic constant or, non-adiabatic behaviour can be used to locate and quantify hidden damage [5].

The unusual mechanical behaviour of near equiatomic NiTi alloys is well reported in the literature, e.g. [6]; however, quantitative experimental stress analysis of components manufactured from this category of material has not yet been demonstrated. The material's complex stress-straintemperature relationship and non-linear mechanical response make some techniques unsuitable and adds significant complexity to the task of interpreting data from other techniques [7].

In the present paper, the feasibility of using TSA to obtain quantitative stress data from a NiTi alloy is considered. The suitability of three loading regions is examined and affect of an intermediate R-phase on the thermoelastic response is investigated in detail. The possibility of using the thermoelastic response to infer information about the material phase change behaviour is investigated and the best approach for experimental analysis of NiTi alloy specimens is discussed. 


\section{Mechanical Behaviour}

The mechanical behaviour of NiTi alloys is strongly dependent on the alloy composition, heat treatment history and mechanical work. It is therefore important to experimentally characterise the as-received test material. Fig. 1 shows a plot of stress versus strain obtained from a tensile test conducted on a $\mathrm{Ni}_{55.8} \mathrm{Ti}_{44.2}$ tube. The tubes were $3 \mathrm{~mm}$ in diameter, with a wall thickness of 0.25 $\mathrm{mm}$, and were cut into specimens of length of $150 \mathrm{~mm}$. The specimen was first loaded and unloaded at a strain rate of $0.025 / \mathrm{s}$, and then loaded from zero stress to failure at the same rate. Testing was conducted using an Instron (Bucks, UK) 8802 static test machine and strain readings were obtained using an extensometer with a $12.5 \mathrm{~mm}$ gauge length. Fig. 1 represents measurements from a single specimen; however, the data are in good agreement with other published work, e.g. [8].

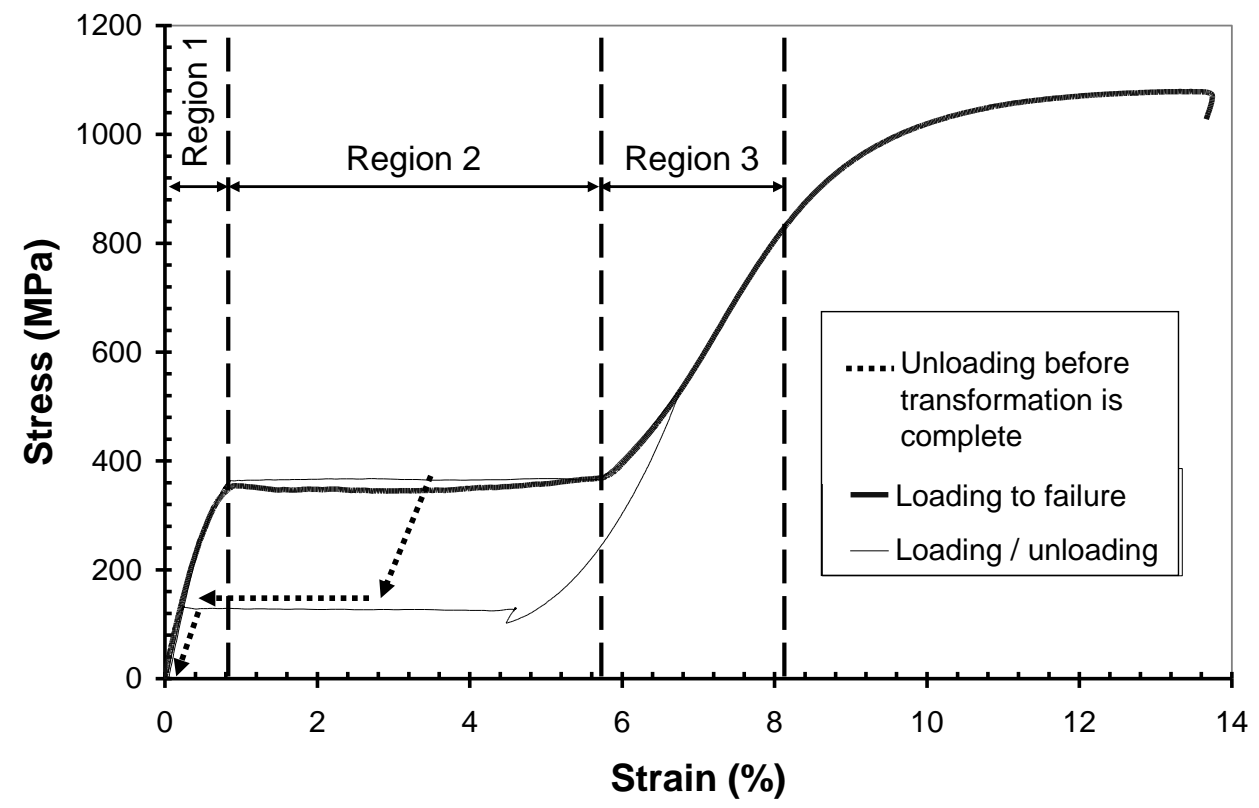

Fig. 1 - Stress-strain properties for NiTi tube specimen

Upon initial loading, the material's microstructure is fully austenitic. The elastic deformation of the austenitic lattice microstructure is represented by the first linear section of the plot, denoted as Region 1 . At approximately $0.8 \%$ strain, the slope of curve reduces to practically zero. This marks the initiation of a stress induced phase transformation to a martensitic microstructure. In this plateau region (denoted as Region 2) the material has little strength and has an inhomogeneous phase distribution. If the specimen is unloaded during the phase transformation, the material responds by first unloading any elastic strain in the martensite phase, before the martensite reverts back to austenite. This behaviour is represented by the non-linear path shown on Fig. 1 by the dotted arrows. The phase transformation to martensite is completed at a strain of $5.6 \%$, where the plateau region ends and the slope of the stress-strain curve increases again. If loading is continued, the martensitic lattice strains elastically until a critical stress is reached, where the material yields and plastic deformation occurs before failure. The martensite region (denoted as Region 3) has a Young's modulus of $16.34 \mathrm{GPa}$ during loading, which increases by $41.5 \%$ to $27.95 \mathrm{GPa}$, when the material is unloaded. If the material is fully unloaded, it returns to its parent austenite phase via a lower transformation plateau as shown in Fig. 1.

Some grades of NiTi alloys transform from the parent austenite structure to martensite via an intermediate phase known as the R-phase [9]. This phase is a slight modification of the parent microstructure characterised by a reduction in the cubic angle, producing a rhombohedral structure. This is manifested in a slight reduction in the slope of the stress-strain curve in Region 1, as shown in Fig. 2. The R-phase content reduces with increasing temperature, with a critical temperature $\left(R_{f}\right)$ above which, it does not occur. 


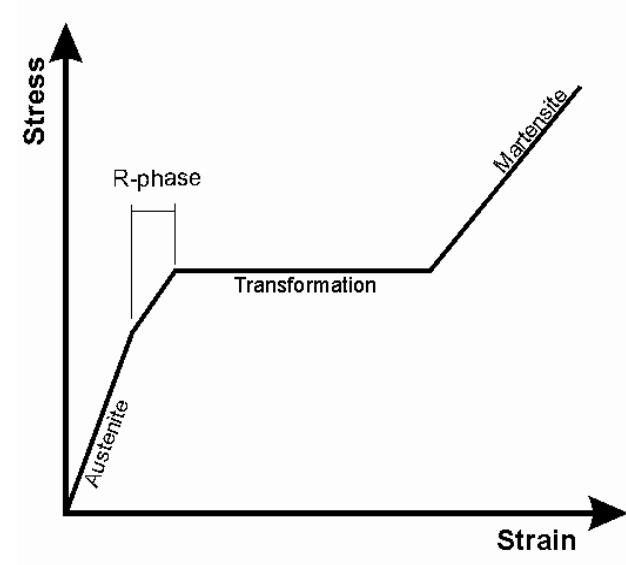

Fig. 2 - Schematic showing mechanical response of NiTi alloy with intermediate $R$ phase

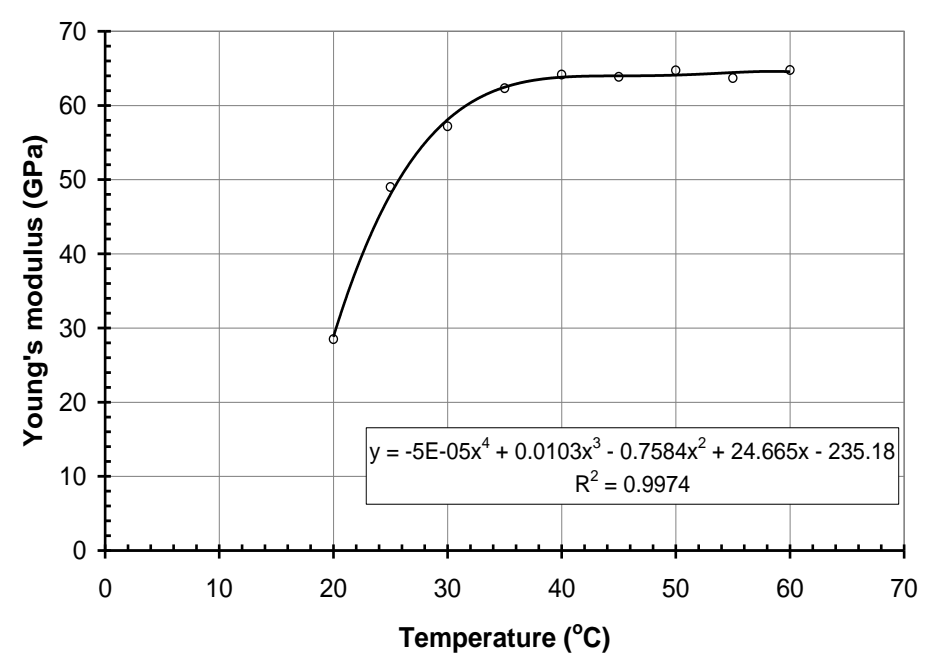

Fig. 3 - Effect of increasing temperature on the mechanical response in Region 1

A series of tensile tests were conducted at increasing temperature using a temperature controlled test chamber [7] to examine the mechanical behaviour of the material at temperatures in the range $20-60^{\circ} \mathrm{C}$. It has been shown that the $R_{S}$ temperature can be quantified mechanically by examining the variation of the Young's modulus with temperature $(\mathrm{d} E / \mathrm{d} T)$ in Region 1 [10]. The results, shown in Fig. 3, demonstrate an increasing trend in the range $20-30^{\circ} \mathrm{C}$. This response is related to the reduction of the R-phase content as the temperature is increased and results in an increase in the Young's modulus in the region. At approximately $40^{\circ} \mathrm{C}$ the plot converges, indicating that a temperature has been reached where the R-phase transformation occurs thermally and therefore is not represented in the stress-strain curve. Above this temperature the Young's modulus is approximately constant, with a value of $64.2 \mathrm{GPa}$ recorded.

The magnitude of the $\mathrm{d} E / \mathrm{d} T$ value is also significant in the analysis of the thermoelastic response. During TSA it is assumed that the thermoelastic response is proportional to the cyclic stress range applied; however, if the $\mathrm{d} E / \mathrm{d} T$ value is large at room temperature the thermoelastic response is a function of the mean stress during cyclic loading. This behaviour makes it impossible to quantify the stresses in a non-uniform stress field [3]. Analysis of Fig. 3 shows that if testing of NiTi specimens is conducted above $40^{\circ} \mathrm{C}$, the $\mathrm{d} E / \mathrm{d} T$ value is $0.017 \mathrm{GPa} /{ }^{\circ} \mathrm{C}$ compared to $4.999 \mathrm{GPa} /{ }^{\circ} \mathrm{C}$ if testing at room temperature. Therefore, the mean stress effect will be significantly reduced and possibly eliminated by testing at $40^{\circ} \mathrm{C}$.

\section{Thermomechanical behaviour}

Each of the three regions identified pose a different challenge for the application of TSA. The phase transformation which occurs in Region 2 is accompanied by significant heating effect during transformation to martensite and cooling when reversed. To examine this behaviour in more detail, variations in the specimen surface temperature were recorded using a FLIR infrared detector (OR, USA). A tube specimen was loaded with a strain rate of $0.25 \times 10^{-3} / \mathrm{s}$ to a stress of $400 \mathrm{MPa}$. The specimen was held at this stress until the specimen temperature had returned to an ambient level, before it was unloaded at the same rate. A typical thermal image of the specimen during loading is shown in Fig 4. The average temperature was recorded in the area indicated. The thermal data was logged at $1 \mathrm{~Hz}$ and correlated with the mechanical response. Fig. 5 shows that an increase in temperature occurs on initial loading. The specimen temperature increases by approximately $2.5^{\circ} \mathrm{C}$ before the onset of martensite formation. Prior to martensite formation there is a reduction in temperature of approximately $1{ }^{\circ} \mathrm{C}$ at a strain of $0.75 \%$. This behaviour is associated with an intermediate phase called the R-phase that will be discussed in more detail in relation to Region 1. 
Martensite formation initiates at a strain of approximately $0.8 \%$. This is accompanied by an increase in temperature. The temperature increases monotonically up to a strain of approximately $4.5 \%$ where the transformation is almost complete. The distribution of heating during the transformation was non-uniform and corresponded to the non-homogenous propagation of the phase transition in the form of a macroscopic helical shear band [11-13]. Loading of the material in the martensite phase does not result in a temperature increase. The temperature variation shown in Fig. 5 for this region shows a steady decay in temperature to the ambient level.

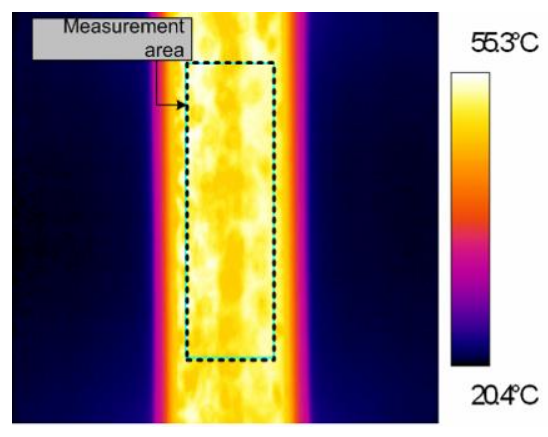

Fig. 4 - Infrared image showing heating in Nitinol tube during martensite formation

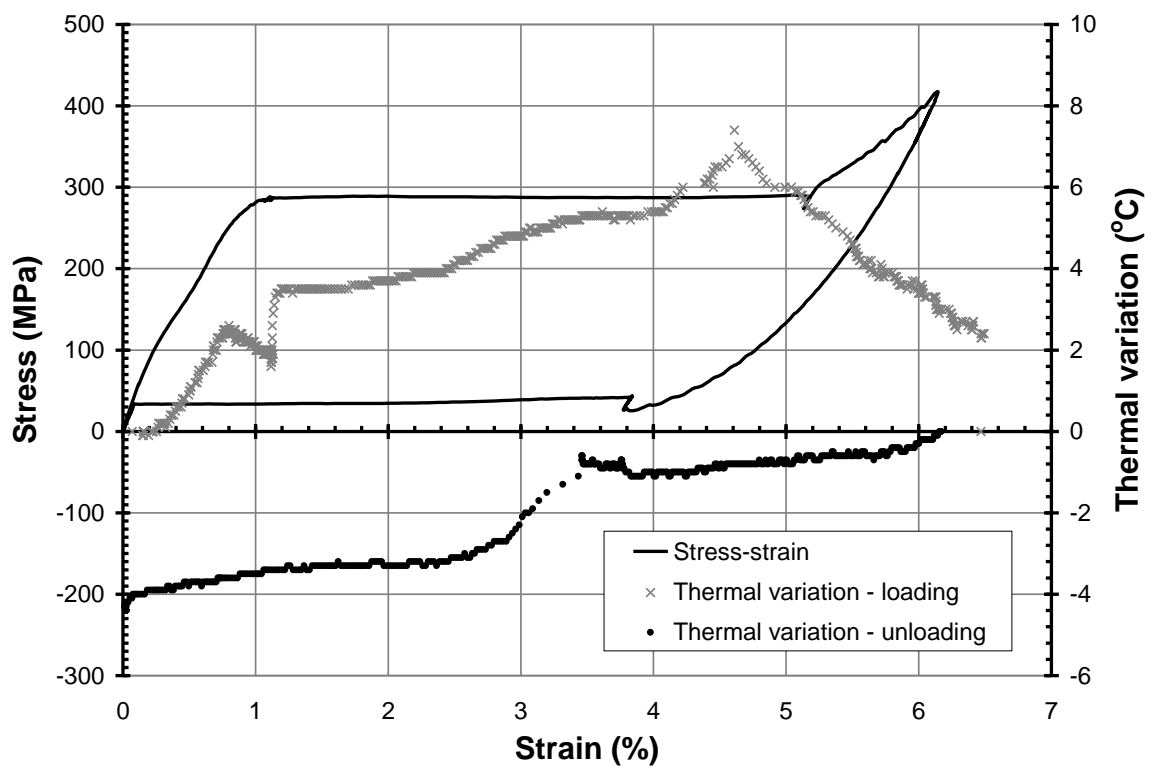

Fig. 5 - Temperature variation in specimen during loading

When unloaded the specimen shows a decrease in temperature of approximately $1^{\circ} \mathrm{C}$ in the range 4 - 6\%, which indicates that a small amount of martensite is being recovered prior to the main transformation plateau. This agrees with the non-linear profile of the curve as it approaches the transformation stress. There is a decrease in temperature of approximately $2.5^{\circ} \mathrm{C}$ when the transformation to austenite is initiated, with a total decrease of approximately $4^{\circ} \mathrm{C}$ over the full reverse transformation. The forward transformation also showed a change in temperature of approximately $4^{\circ} \mathrm{C}$.

It has been confirmed that significant temperature variations occur during the phase transformations in Nitinol. The magnitude and inhomogeneous distribution of these variations poses a significant challenge for TSA. In addition, the low Young's Modulus in this region makes load controlled cyclic loading unpractical.

Obtaining functional thermoelastic measurements from Region 3 is made difficult by two main factors. Firstly, there is a significant hysteresis effect evident when the material is cyclically loaded in this region; TSA requires linear elastic loading. Secondly, preliminary testing showed that the thermoelastic signal was mean stress dependent in this region. Test were carried out to characterise the $\mathrm{d} E / \mathrm{d} T$ value in this region [14], however, it was not possible to account for the mean stress effect theoretically. It was concluded that a more detailed analysis of the microstructural behaviour was required to understand the thermoelastic response and that currently Region 3 is not suitable for TSA. 


\section{Quantitative Stress Analysis}

The material's Young's modulus in Region 1 is temperature dependent at room temperature and theoretically should result in mean stress dependence of the thermoelastic response. In order to confirm this, calibration type tests were carried out at room temperature on the tensile specimens manufactured from the tubes of the material. The use of a tensile specimen effectively eliminates any non-adiabatic behaviour so it was possible just to observe the effect of increasing the mean stress whilst maintaining a constant stress range. Further details of the experimental arrangement and theoretical calculations can be found in [7].

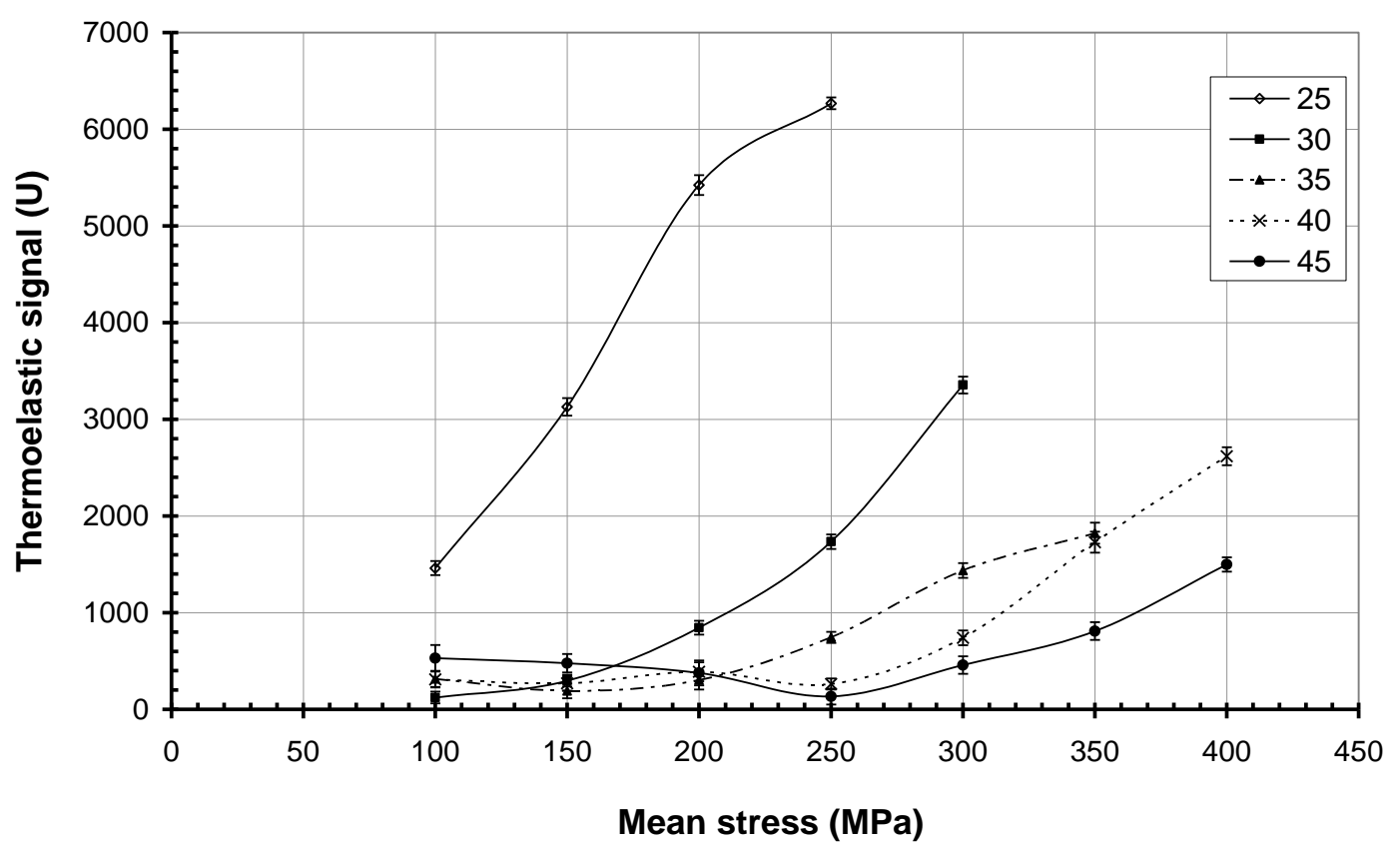

Fig. 6 - Mean stress dependence in austenite region recorded at increasing temperatures

To confirm experimentally the theoretical predication that the mean stress effect can be minimised by testing at $40^{\circ} \mathrm{C}$, an analysis was conducted at a range of elevated temperatures using a specially constructed test chamber [7]. Using the chamber it was possible to control the specimen temperature to an accuracy of $\pm 1{ }^{\circ} \mathrm{C}$. The thermoelastic response of a specimen was examined over a range of mean stresses within Region 1 , at temperatures in the range $25-45^{\circ} \mathrm{C}$. The results of the test are shown in Fig. 6. The thermoelastic signal recorded at $25^{\circ} \mathrm{C}$ shows that at room temperature the material response is highly dependent on the mean stress. The curve obtained at $30^{\circ} \mathrm{C}$ shows evidence of a reduction in the dependence. This is encouraging, as it indicates that the mean stress dependence reduces with temperature. The trend is continued as the temperature is increased to $35^{\circ} \mathrm{C}$, with the magnitude of the data again decreasing and the slope of the plot showing a further decrease of more than $50 \%$. The data obtained at 40 and $45^{\circ} \mathrm{C}$ shows an approximately uniform response in the range $100-250 \mathrm{MPa}$. However, the signal obtained at these temperatures was low in magnitude and contained considerable noise. The mean stress dependence reappears at mean stresses above $250 \mathrm{MPa}$. This effect may be related to the proximity of the loading to the region where the material begins to transform to martensite. This work demonstrates that under certain conditions the mean stress dependence of the thermoelastic response obtained from Nitinol can be eliminated. However, further work is required to investigate if the magnitude and reliability of the signal can be improved.

From the data of the type shown in Fig. 6 it was possible to determine a calibration constant for the Nitinol at $40^{\circ} \mathrm{C}$ of $0.325 \mathrm{MPa} / \mathrm{U}$ using the average signal obtained from the area shown in Fig. 4 from data collected with mean stresses in the range $100-250 \mathrm{MPa}$. The variation in this data is approximately $25 \%$, which can be attributed to the low response obtained at this temperature. It may be possible to improve the signal to noise ratio of the data by increasing the stress range 
applied during loading. However, this will introduce new difficulties as part of the cycle will enter the transformation region, resulting in mean stress dependence.

\section{Conclusions}

The practicality of TSA for quantitative stress analysis of non-uniform stress fields in NiTi alloys was considered. It was identified that transformation region are not suitable for the application for TSA due to significant inhomogeneity and heating effects. The effects of hysteresis loading and variations in the Young's modulus in the martensitic region make the application of TSA currently, unpractical. It was identified that quantitative stress analysis can be conducted in the pre-martensite phase, if testing is conducted at a temperature where the R-phase does not occur and temperature dependence of the material's Young's modulus is minimised.

\section{Acknowledgements}

Financial support provided by the Irish Research Council for Science, Engineering and Technology and equipment loan from the UK Engineering and Physical Science Research Council loan pool is graciously acknowledged

\section{References}

1. $\quad$ Dulieu-Barton, J.M.: Strain Vol. 35 (1999), p. 35-39.

2. $\quad$ Stanley, P. and Chan, W.K.: J. of Strain Analysis Vol. 20 (1985), p. 129-137.

3. Wong, A.K., Jones, R., and Sparrow, J.G.: J. of Phys. Chem. Solids Vol. 48 (1987), p. 749753.

4. $\quad$ Quinn, S., Dulieu-Barton, J.M., and Langlands, J.M.: Strain Vol. 40 (2004), p. 127-133.

5. Sathon, N. and Dulieu-Barton, J.M.: Applied Mechs. and Mats. Vol. 7-8 (2007), p. 153-158.

6. Duerig, T.W.: Engineering Aspects of Shape Memory Alloys (Butterworth-Heinemann, London 1990).

7. Dulieu-Barton, J.M., Eaton-Evans, J., Little, E.G., and Brown, I.A.: Strain Vol. 44 (2008), p. 102-118.

8. Sun, Q.-P. and Li, Z.-Q.: International Journal of Solids and Structures Vol. 39 (2002), p. 3797-3809.

9. $\quad \mathrm{Ng}$, K.L. and Sun, Q.P.: Mechanics of Materials Vol. 38 (2006), p. 41-46.

10. Eaton-Evans, J., Dulieu-Barton, J.M., Little, E.G., and Brown, I.A.: J. of Mech. Eng. Sci. Vol. 222 (2008), p. 97-105.

11. Pieczyska, E.A., Gadaj, S.P., Nowacki, W.K., and Tobushi, H.: Exp. Mechs. Vol. 46 (2006), p. 531-542.

12. Shaw, J.A. and Kyriakides, S.: Intl. J. of Plasticity Vol. 13 (1997), p. 837-871.

13. Sittner, P., Liu, Y., and Novak, V.: J. of Mech. and Phys. of Solids Vol. 53 (2005), p. 17191746.

14. Eaton-Evans, J., Dulieu-Barton, J.M., Little, E.G., and Brown, I.A.: J. of Strain Analysis Vol. 41 (2006), p. 481-495. 